\title{
La photo et la carte: l'exemple de Moutier et ses gorges
}

Suivant les fragments des cartes officielles et la carte générale de la Suisse, l'Atlas mondial suisse présente une page peu commune. En effet, le début des cartes régionales est consacré au thème de la cluse de Moutier (page 6), avec deux documents insolites: une photo aérienne oblique et une carte topographique au $25000 \mathrm{e}$ avec le développement de deux thèmes: l'utilisation du sol en quatre catégories et la coloration stratigraphique de trois couches de roches affleurantes.

Par sa combinaison de deux types de représentations, cette page offre trois domaines d'application: la cartographie et la télédétection, la géographie thématique et la géographie régionale.

\section{Photo oblique et carte topographique: une comparaison}

Si dans certains atlas, les illustrations photographiques sont nombreuses (ex. JEANNERET et AUF DER MAUR 1982), l'Atlas mondial suisse est essentiellement une collection de cartes géographiques et thématiques avec quelques exemples de documents de télédétection. Ces derniers ont avant tout une fonction didactique, ils permettent une appréciation de ce type de représentation spatiale, mais ne constituent pas un élément systématique - à même titre que les paires de cartes physiques et économiques par exemple.

En principe, deux approches comparatives sont possibles à partir des deux documents présentés: comparaison entre la vue oblique et la vue plongeante, et entre la photo et la carte. En effet, la photo de Moutier est l'unique document de ce type de l'Atlas, et le seul offrant une vue oblique. Elle servira à démontrer les démarches nécessaires pour passer d'une vue familière à l'abstraction qu'exige la vue plongeante - un pas plus difficile à franchir que celui menant de la photo ou l'image plongeante à la carte (MARR 1985).

Des lignes rouges sur la carte définissent le champ de vision approximatif de la vue aérienne prise à environ $700 \mathrm{~m}$ de hauteur, donc à $1200 \mathrm{~m}$ d'altitude. On constate qu'à l'arrière-plan, des parties du paysage sont dissimulées par des obstacles orographiques. Ainsi, Les Maisons Dessus de Roches sont visibles sur la photo, contrairement au bas du village, caché derrière l'Arête du Raimeux. Voilà l'occasion de démonter la valeur des représentations plongeantes, leur optique étant universelle, tout y est en principe visible. Mais voilà le prix d'une abstraction pas évidente pour le lecteur de carte peu habitué. Ensuite, il y a le rôle de l'échelle dans les deux cas: la comparaison de quelques distances équivalentes, sur la photo et la carte, permet d'apprécier les spécificités des conditions géométriques. Ainsi, les distances entre la ferme Sur Chaux et le clocher de l'eglise catholique moderne, en ville, entre la Birse et la voie de chemin de fer, et entre les points culminants de la première cluse (Les Golats, cote $835 \mathrm{~m}$, et La Maisonnette, $964 \mathrm{~m}$ ) peuvent être comparées. Les deux distances d'environ $1 \mathrm{~km}$ chacune correspondent, sur la photo, à 13 respectivement $10 \mathrm{~cm}$. Ceci signifie que l'échelle d'une vue oblique est variable, grande à l'avant-plan et petite à l'arrière-plan.

On découvrira que la vue oblique permet de voir plus loin, puisqu'à l'arrière-plan à droite, les parois rocheuses de Montchemin sur Choindez sont visibles, tandis que l'on devine quelques éléments du bassin de Delémont tout à l'arrière. Les techniques d'impression choisies pour l'Atlas mondial suisse sont optimales pour les cartes, mais les photos ne sont malheureusement pas rendues dans la même qualité.

\section{Vallées jurassiennes longitudinales et transversales}

La page de l'Atlas consacrée à Moutier n'est pas le seul exemple du Jura plissé: la vallée de Joux est aussi représentée par une carte thematique (Atlas p. 8), comme le pied du Jura avec Crissier (p. 16). Le cas de Moutier permet de développer quelques spécificités, plus ou moins caractéristiques du Jura. La situation peut être qualifiée d'encaissée, mais dispose de l'avantage de la confluence de plusieurs vallées: celles de la Birse (nord-sud), de La Chalière de l'ouest et de la Raus de l'est, encadrée par la montagne de Moutier et Raimeux au nord et Mont-Girod et Graitery au sud, des chaines culminant entre 1000 et $1300 \mathrm{~m}$. La carte accuse à différents endroits les effets de l'instabilité des versants sud, reconnaissable par le tracé des courbes de niveau.

François Jeanneret, Dr, Université de Berne, Institut de Géographie, Hallerstraße 12, 3012 Berne 
Moutier est donc caractérisée par la croisée d'une vallée longitudinale avec deux vallées transversales. La première comporte deux tronçons courts: le Grand-Val d'environ $7 \mathrm{~km}$ à l'est, le Petit-Val d'environ $4 \mathrm{~km}$ à l'ouest. La carte de la page 6 de l'Atlas ne permet pas d'apprécier cette situation, tandis que la carte générale de la Suisse (page 4) est trop généralisée dans ce cas. Une carte murale ou une carte nationale (1106 Moutier ou 223 Delémont) offrent une vue plus générale. En classe, l'affichage d'une de ces cartes s'imposera. Car en effet, cinq voies de communications convergent sur Moutier:

- la vallée de la Birse (aval): Bâle-Delémont-Choindez (CFF et Transjurane),

- vallée de la Raus: Balsthal-Gänsbrunnen et Soleure (SMB et route),

- vallée de la Chalière: Bellelay-Sornetan-Souboz (route),

- vallée de la Birse (amont): Bienne-vallée de Tavannes-Court (CFF, route et future Transjurane),

- Granges par le tunnel de la montagne de Granges (ferroviaire uniquement, BLS).

\section{Les gorges de Moutier:}

\section{des cluses pas comme les autres}

Les gorges de Moutier sont probablement les cluses jurassiennes les plus souvent présentées dans la littérature géographique. Ceci pour différentes raisons: les formes sont très distinguées, les dimensions impressionnantes, les environs habitées, les voies de communication importantes et très fréquentées, les rochers sont recherchés par les varappeurs. Les gorges de Moutier sont donc très connues. Sans vouloir évoquer tous les facteurs pouvant participer à la genèse des cluses, cités par MONBARON (1975), constatons qu'il n'est pas question de se limiter à un seul (par exemple l'antécédence, voir BÄR 1988: 25, BURRI 1995: 27). La figure 1 présente un relevé des cluses du Jura central. L'alignement de nombreuses cluses de cette région apparaît clairement. PORTMANN (1991 et 1995) présente une analyse détaillée de la géomorphologie de la région de Moutier, tandis que les amateurs de géologie trouveront une description détaillée et les coupes classiques dans des guides (p. ex. LAUBSCHER 1967).

Pourtant, les cluses de Moutier sont aussi très particulières: elles sont peu inclinées (pour des cluses du Jura plissé), traversent plusieurs synclinaux, accusent des dimensions exceptionnelles, sont peu praticables autrement qu'en train ou en voiture (il n'existe pas de chemin pédestre ou piste cyclable).

\section{L'habitat: quelques observations}

Sur le plan de la géographie humaine, la photo aérienne est dominée par la ville de Moutier. Pourtant, une partie seulement de la ville est visible, dont la vieille ville, située

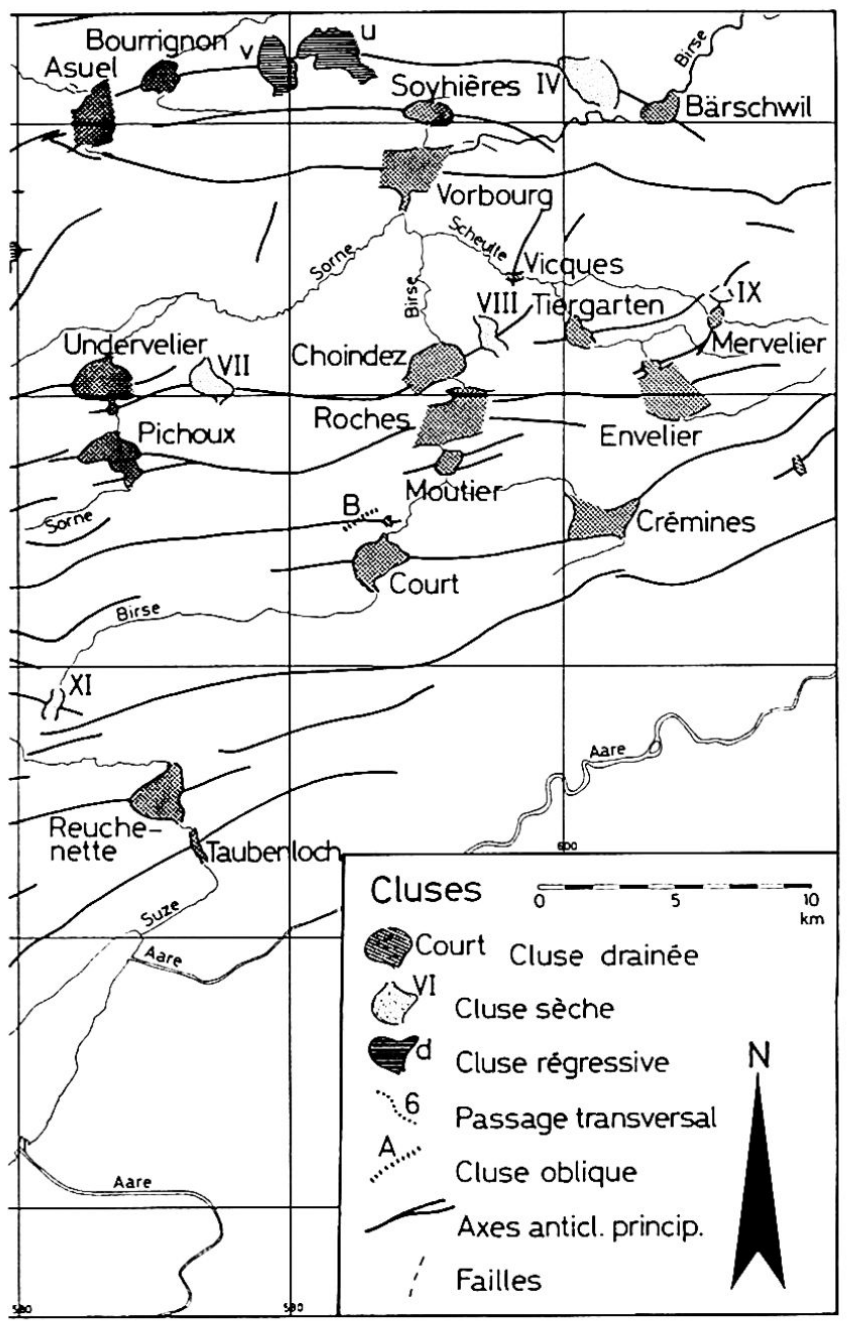

Fig.1 Les cluses du Jura central. Extrait de MONBARON 1975, annexe VI.

au pied du versant. De grands volumes frappent l'observateur: l'hôpital sous la forêt des Golates, puis différents immeubles du centre-ville, en particulier autour de la gare. Ces constructions relativement récentes contrastent par leur couleur claire avec les grands volumes moins hauts de l'ancienne industrie. A l'est de la gare on discerne une des premières usines de l'industrie clé de Moutier: la production des tours automatiques. Les bâtiments actuels se trouvent cependant à l'ouest, le long de la route de Court, on les découvre sur la carte.

Au XIXe siècle, Moutier s'ouvrit à l'industrie. Depuis le début du siècle, l'horlogerie commença à s'implanter dans le bourg. Une verrerie fut ouverte dans le grand village en 1840, unique en Suisse, à grande renommée. Peu à peu, la mécanique de précision fit son apparition et donna à Moutier un nouvel essor. Le développement de industrie est plus récent que dans d'autres localités et correspond à un aménagement différent. L'industrie mécanique nécessite des surfaces plus importantes que l'horlogerie, elles se développent de préférence à l'extérieur de la ville. 
La deuxième localité visible sur la photo se trouve à l'arrière-plan, dans une des cluses des gorges de Moutier: Roches (Les Maisons Dessus). Souvent les centres de cluses suffisamment éventrées sont occupés par un village. Si cet emplacement nous semble actuellement défavorisé par l'encaissement, par les inconvénients climatiques (ombragé, fréquence élevée d'inversions thermiques et de brouillard) et topographiques, il y a aussi des avantages incontestables: dans le passé, souvent les affleurements de minerais furent la base d'une industrialisation parfois importante (Choindez à l'arrière-plan), et les centres de services le long des voies de communication desservent l'habitat rural avoisinant.

\section{Propositions de travaux pratiques}

Toute une série de travaux pratiques sont réalisables à partir de la page 6 de l'Atlas mondial suisse, consacrée à Moutier et ses cluses. La liste suivante comporte un nombre de suggestions de thèmes à développer.

Problèmes de cartographie et de télédétection:

- Comparaison de distances équivalentes sur les deux documents

- Repérer des éléments sur la photo aérienne à l'aide de la carte de la page 6 ou de la carte nationale 223 Delémont

- Vérification du relevé de l'utilisation du sol

- Comparaison de l'état des deux documents

- Comparaison de la photo de l'Atlas avec d'autres photos aériennes de Moutier (par ex. BÄR 1988: 25)

- Comparaison d'une photo aérienne avec une image de satellite (par ex. Tessin, page 21 de l'Atlas)

Problèmes thématiques:

- Description de la disposition des couches géologiques

- Définition d'une cluse

- Répartition de la forêt

- Localisation des pâturages communs

- Discussion du tracé de la route et du chemin de fer

- Appréciation du site des villages dans les cluses

D'autres documents pourraient compléter la page de l'Atlas (cartes, manuels, photos, le tableau scolaire suisse no 184). Les thèmes proposés seront adaptés au but à suivre et à la situation scolaire.
L'Atlas mondial suisse est sans doute un prestigieux moyen d'enseignement pour la géographie (JEANNERET 1993). Dans cet ouvrage, la page "Cluse de Moutier» est exceptionnelle: elle offre un accès au langage cartographique, elle permet la démonstration de moyens de télédétection et elle contribue à la géographie régionale d'une région fascinante, le Jura plissé.

\section{Bibliographie}

BÄR, O. (1988): Géographie de la Suisse, Delcourt Lausanne, $246 \mathrm{p}$.

BURRI, K. (1995): Schweiz Suisse Svizzera Svizra, Geographische Betrachtungen, Interkantonale Lehrmittelzentrale, Lehrmittelverlag des Kantons Zürich, $338 \mathrm{p}$.

JEANNERET, F. (1993): L'Atlas mondial suisse: une ancienne tradition et une nouvelle édition. Dans: Bulletin de la Société neuchâteloise de géographie 37, p. 7-16.

JEANNERET, F., AUF DER MAUR, F. (1982): Grand Atlas de la Suisse, Kümmerly + Frey, Berne, et Editions 24 heures, Lausanne, $208 \mathrm{p}$.

LAUBSCHER, H.P. (1967): Basel-Delémont-Moutier-Biel. Exkursion Nr. 14, Geologischer Führer der Schweiz - Guide géologique de la Suisse, Heft 4, Wepf Basel, 217-237.

MARR, R. L. (1985): Schweizer Weltatlas, Kommentarband zur Ausgabe 1981, Schweizerische Konferenz der kantonalen Erziehungsdirektoren, Zurich, $40 \mathrm{p}$.

MONBARON, M. (1975): Contribution à l'étude des cluses du Jura septentrional. Thèse Fac. sc., Université de Neuchâtel, 208 p. et 8 planches.

PORTMANN, J.-P. (1991): Analyse géomorphologique de la carte "Moutier". Dans: Bulletin de la Société neuchâteloise de géographie 35/Jahrbuch der Geographischen Gesellschaft Bern 57, 11-24.

PORTMANN, J.-P. (1995): Paysages de Suisse: Le Jura. Introduction à la géomorphologie. Geographica Bernesia P 24, Institut de géographie de l'Université de Berne, $152 \mathrm{p}$.

TABLEAU SCOLAIRE SUISSE = SCHWEIZER SCHULWANDBILD "Cluse de Moutier", no 184, Ingold, Herzogenbuchsee. 


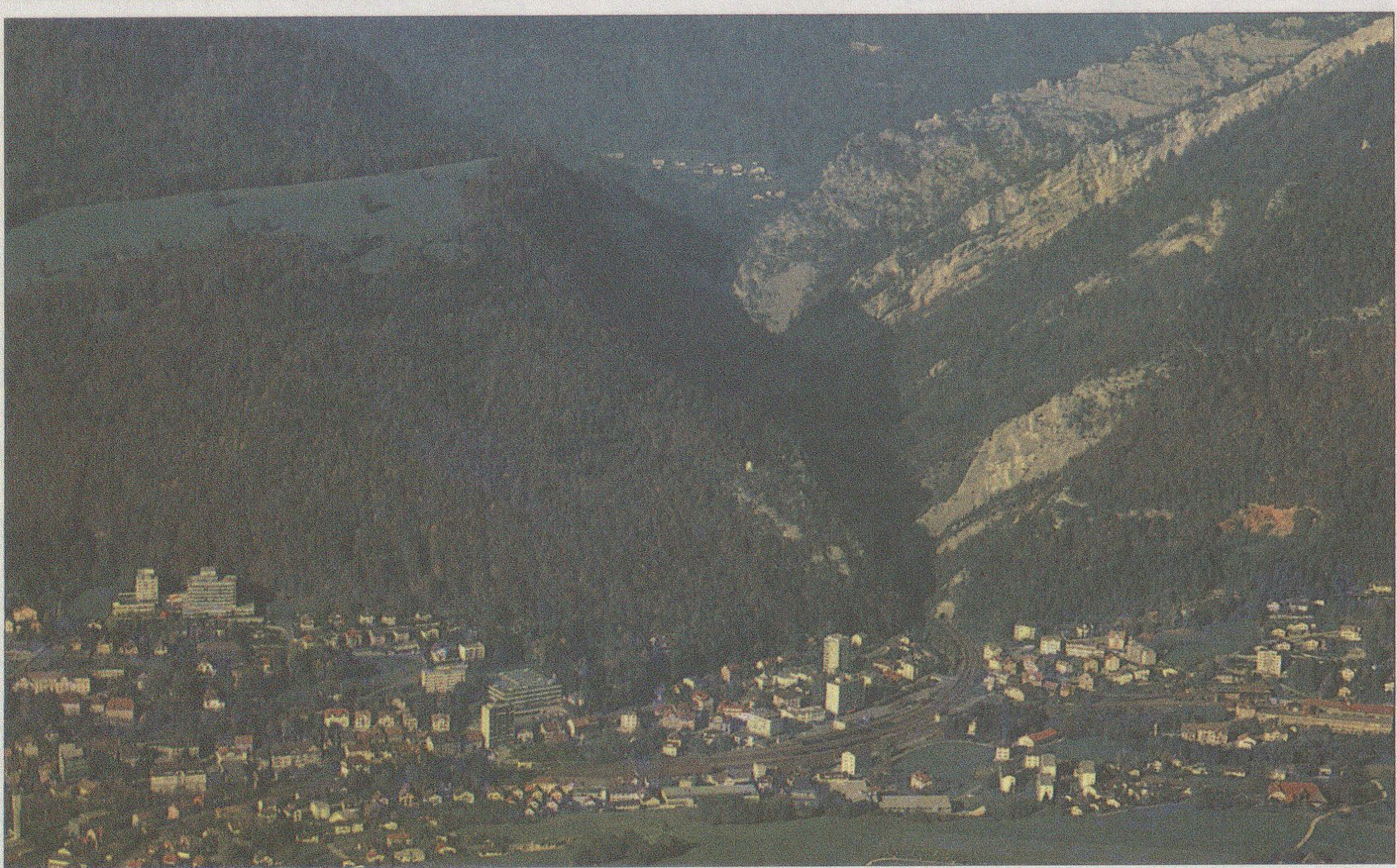

\section{Cluse de Moutier}

Photo aérienne oblique du sud

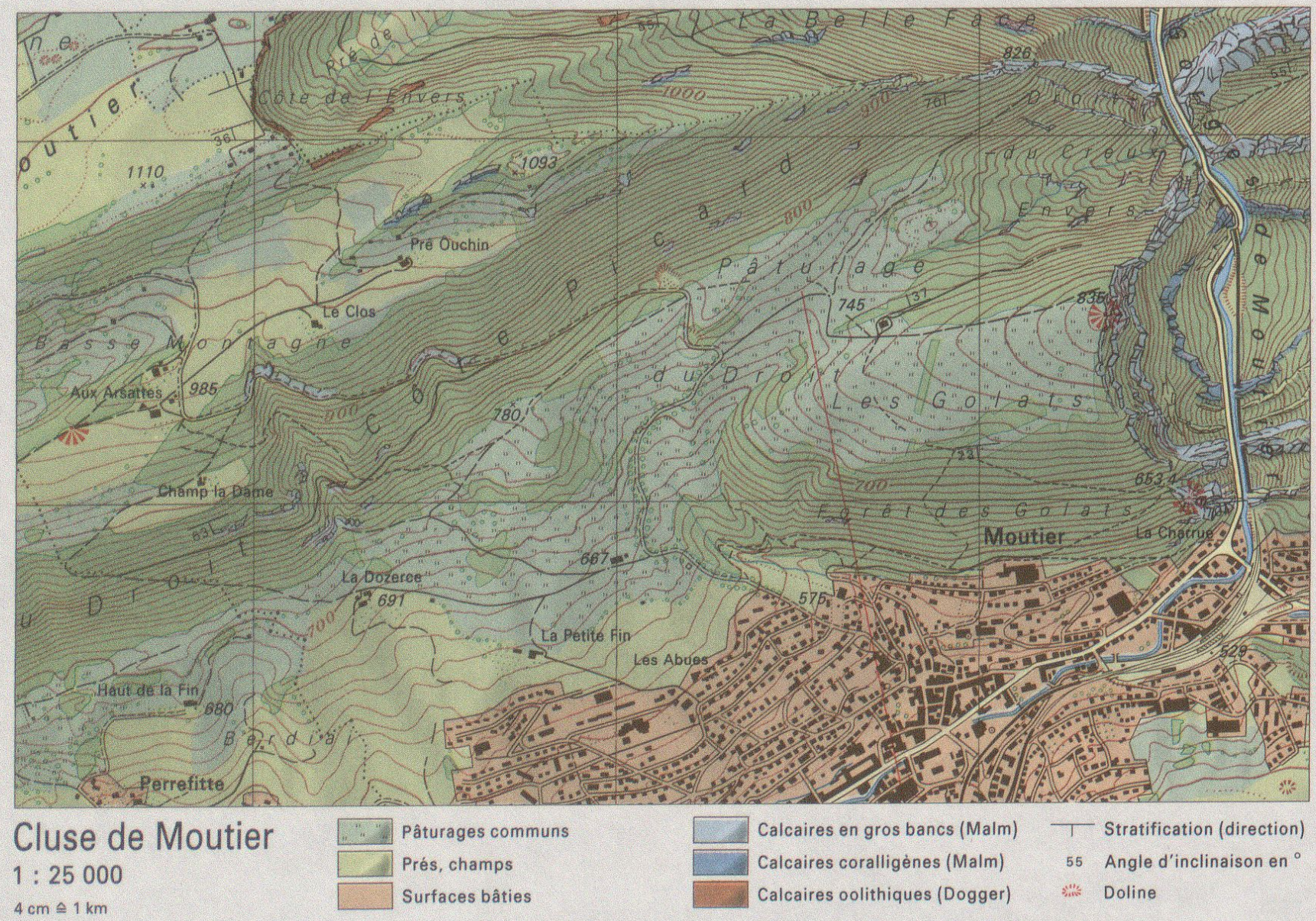

